\title{
Structure and Parametrization of Stochastic Maps of Density Matrices
}

\author{
E.C.G. Sudarshan \\ Department of Physics and Center for Particle Physics \\ University of Texas, Austin, Texas 78712-1081
}

\begin{abstract}
The generic linear evolution of the density matrix of a system with a finite-dimensional state space is by stochastic maps which take a density matrix linearly into the set of density matrices. These dynamical stochastic maps form a linear convex set that may be viewed as supermatrices. The property of hermiticity of density matrices renders an associated supermatrix hermitian and hence diagonalizable; but the positivity of the density matrix does not make this associated supermatrix positive. If it is positive, the map is called completely positive and they have a simple parametrization. This is extended to all positive (not completely positive) maps. A contraction of a norm-preserving map of the combined system can be contracted to obtain all dynamical maps. The reconstruction of the extended dynamics is given.
\end{abstract}

*e-mail: sudarshan@physics.utexas.edu 


\section{Introduction: Dynamical Maps}

The Quantum Density Matrix $\rho$ is the statistical state and therefore quantum dynamics is the evolution of the density matrix. For a closed system this evolution is by a unitary time-dependent operator:

$$
\rho(t)=U(t) \rho(0) U^{\dagger}(t)
$$

The evolution is linear. But if we have an open system, its dynamics cannot be by a unitary evolution but a more general linear evolution[1]:

$$
\rho(t)=A(t) \rho(0)
$$

where $A$ is a linear map of the density matrix into a density matrix. This superoperator $A(t)$ can be written as a supermatrix.

$$
\rho_{r, s}(0) \longrightarrow A_{r s, r^{\prime} s^{\prime}}(t) \rho_{r^{\prime} s^{\prime}}(0)=(A(t) \rho)_{r s}
$$

Then the supermatrix $A(t)$ must satisfy the following three constraints:

$$
\begin{aligned}
A_{s r, s^{\prime} r^{\prime}}(t) & =A_{r s, r^{\prime} s^{\prime}}(t) \\
\rho(0) \geq 0 & \longrightarrow A_{r s, r^{\prime} s^{\prime}} \rho_{r^{\prime} s^{\prime}} \geq 0 \\
A_{s s s, r^{\prime} s^{\prime}} & =\delta_{r^{\prime} s^{\prime}} .
\end{aligned}
$$

These are consequences of the hermiticity, positivity and trace properties of the density matrices. These properties can be best expressed in terms of the dynamical matrix[2].

$$
\begin{aligned}
& B_{r r^{\prime}, s^{\prime} s}(t)=A_{r s, r^{\prime} s^{\prime}}(t) \\
& B_{s^{\prime} s, r r^{\prime}}^{*}(t)=B_{r r, s^{\prime} s}(t) \\
& \quad x_{r}^{*} y_{r}, B_{r r^{\prime}, s^{\prime} s}(t) x_{s} y_{s^{\prime}}^{*} \geq 0 \\
& B_{n r^{\prime}, s^{\prime} n}(t)=\delta_{r^{\prime} s^{\prime}} .
\end{aligned}
$$


Thus $B$ is a hermitian matrix which gives nonnegative expectation value for supervector that can be factorized:

$$
u^{\dagger} B u \geq 0 \text { if } u_{r s}=x_{r} y_{s}^{*} .
$$

It is sufficient if $B$ is nonnegative but it is not necessary. If $B \geq 0$, then we will call the map "completely positive;" [2] but if only the positivity condition (4) is satisfied, we will call the map "positive but not completely positive". Since $B$ is hermitian, according to (4) it follows that it has an eigenvector decomposition

$$
B_{r r^{\prime}, s^{\prime} s}=\sum \mu_{\alpha} \zeta_{r r^{\prime}}^{(\alpha)} \zeta^{*(\alpha)}=\zeta M \zeta^{\dagger}
$$

when $M$ is the diagonal matrix with eigenvalue $\mu_{\alpha}$, and $\zeta_{r r^{\prime}}^{(\alpha)}$ are the normalized eigenvectors. For a completely positive map all the $\mu_{\alpha}$ are nonnegative, but this will not be true for not completely positive maps. If all the $\mu_{\alpha}$ are nonnegative, we can absorb them by defining the eigenvector

$$
C_{r r^{\prime}}^{(\alpha)}=\mu_{\alpha}^{1 / 2} \zeta_{r r^{\prime}}^{(\alpha)}
$$

So for, a completely positive map[2]

$$
\rho \longrightarrow \sum_{\alpha} C^{(\alpha)} \rho C^{(\alpha) \dagger}
$$

with the trace condition

$$
\sum_{\alpha} C^{(\alpha) \dagger} C^{(\alpha)}=1
$$

\section{Not Completely Positive Maps}

For a not completely positive map, some eigenvalues $\nu$ are negative. We may then define

$$
\rho \longrightarrow \sum_{\alpha} C^{(\alpha) \dagger} \rho C^{(\alpha)}-\sum_{\beta} D^{(\beta) \dagger} \rho D^{(\beta)}
$$

with

$$
D_{r r^{\prime}}^{(\beta)}=(|\nu|)^{1 / 2} \eta_{r r^{\prime}}^{\beta} .
$$


It will turn out that the number of negative eigenvalues cannot exceed the number of positive eigenvalue. The trace condition now becomes

$$
\begin{gathered}
\sum_{\alpha} C^{(\alpha) \dagger} C^{(\alpha)}-\sum_{\beta} D^{(\beta) \dagger} D^{(\beta)}=1 . \\
\sum_{\alpha} C^{\alpha} u_{\alpha} u_{\alpha}^{\dagger} C^{\alpha \dagger}-D^{\beta)} u_{\beta} u_{\beta}^{\dagger} D^{(\beta) \dagger} \geq 0 .
\end{gathered}
$$

In dealing with maps we have the convexity property:

$$
B^{I} \cos ^{2} \theta+B^{I I} \sin ^{2} \theta=B
$$

is a positive map if $B^{I}$ and $B^{I I}$ are positive maps. Every map that cannot be expressed in this manner is called extremal:

$$
B=B^{I} \cos ^{2} \theta+B^{I I} \sin ^{2} \theta \longrightarrow B^{I} \equiv B^{I I}
$$

If we can determine all extremal maps, we can generate all maps from them.

Since the density matrices are restricted by positivity and the trace condition, they form a compact set. The dynamical maps map from a compact set into a compact set, the maps also form a compact set.[1] Such a set can be generated from its extremal elements. Then

$$
\sum_{\alpha} \operatorname{tr}\left(C^{(\alpha)} C^{\dagger}\right)-\sum_{\beta} \operatorname{tr}\left(d^{\beta)} d^{(\beta) \dagger}\right)=N
$$

Define

$$
\sum_{\alpha} C^{(\alpha)} C^{(\alpha) \dagger}=J \geq 0, \sum_{\beta} D^{(\beta) \dagger}=K \geq 0 .
$$

We can do unitary transformations $U, V$ on the initial and final density matrices we can diagonalize $J$. By virtue of (11) this will diagonalize $K$. Then

$$
J-K=1
$$

If the eigenvalues of $K$ are $k_{\alpha}^{2}$ and for $J$ they are $j_{\alpha}^{2}$, then

$$
k_{\alpha}^{2}=j_{\alpha}^{2}+1 .
$$


Define $\vartheta_{\alpha}$ so that

$$
j_{\alpha}=\sinh \vartheta_{\alpha}, k_{\alpha}=\cosh \vartheta_{\alpha}
$$

Define

$$
\begin{aligned}
C^{(\alpha)} & =\cosh \vartheta_{\alpha} M(\alpha) \\
D^{(\alpha)} & =\sinh \vartheta_{\alpha} N(\alpha)
\end{aligned}
$$

so that

$$
\sum_{\alpha=1}^{m} M_{(\alpha)} M_{(\alpha)}^{\dagger}=1, \sum_{\beta=1}^{n} N(\beta) N^{\dagger}(\beta)=1 .
$$

Parametrizising these matrices is already known[3]. In those cases where $\sinh \vartheta_{\alpha}=0$, the matrix $N(\alpha)$ are not defined and we sum over a smaller set than the set of $M(\alpha)$.

Choose a unitary transformation $W_{1}$ so that $M_{(1)}^{1}$ is diagonal with eigenvalues $\cos \theta_{1}^{(1)}$ :

$$
W_{1}^{\dagger} M(1) W_{1}=\left(\begin{array}{ccc}
\cos \theta_{1}^{(1)} & & 0 \\
& \cos \theta_{2}^{(1)} & \\
0 & & \ddots \cos ^{2} \theta_{m}^{(1)}
\end{array}\right)
$$

Than

$$
W_{1}^{\dagger} \sum_{2}^{m} C^{(\alpha)} C^{(\alpha) \dagger} W_{1}=\left(\begin{array}{ccc}
\sin ^{@} \theta_{1}^{(1)} & & 0 \\
& \sin ^{2} \theta_{2}^{(1)} & \\
0 & & \ddots \sin ^{2} \theta_{n}^{(1)}
\end{array}\right)
$$

Define

$$
M_{(\alpha)}^{(2)}=\left(\begin{array}{ccc}
\sin \theta_{1}^{(1)} & & 0 \\
& \sin \theta_{2}^{(1)} & \\
& & \ddots \sin \theta_{N}^{(1)}
\end{array}\right) M(\alpha), 2 \leq \alpha \leq N .
$$

Then define the $(N-1) \times(N-1)$ matrices which satisfies

$$
M^{(2)}(m) M^{(2) \dagger}(m)=1_{N-1 \times N-1}
$$

We now repeat the procedure. Define

$$
W_{2}^{\dagger} M^{(2)}(2) W_{2}=\left(\begin{array}{ccc}
\cos \theta_{1}^{(2)} & & 0 \\
& \cos \theta_{2}^{(2)} & \\
& 0 & \ddots \cos \theta_{m}^{(2)}
\end{array}\right)
$$


and a new orthogonal transformation $\sigma_{\alpha}^{(2)}$ such that $\theta_{2}^{(2)}=0$ and we can define $M_{(m)}^{(2)}$ so that we can introduce the $m-2$ matrices $M^{(2)}(m)$ which satisfy

$$
\begin{aligned}
M^{(2)}(m) & =\left(\begin{array}{ccc}
\cos \theta_{1}^{(3)} & & 0 \\
& \cos \theta_{2}^{(3)} & \\
0 & & \ddots \cdot \cos ^{(3)} \theta_{3}
\end{array}\right) \\
\left.\sum M_{(}^{(3)} m\right) M_{(m)}^{(3) \dagger} & =1_{N, N}
\end{aligned}
$$

This procedure can be carried out until all matrices are parametrized. The $M_{x}$ needs $m$ parameter $\theta_{1}^{(1)} \ldots, \theta_{m}^{(1)}$ and so on until we get, a total of $m^{2}$ angles. The unitary matrices are also relevant parameters which determine the dynamical map.

The same procedure can be carried out to parametrize $K$. We need $n^{2}$ angles. Together then, the matrices $J, K$ together require angles $\vartheta_{1} \ldots, \vartheta_{m}, m^{2}$ angles for $J, n^{2}$ angles $K$; a total of $\left(m^{2}+n^{2}\right)$ parameters to determine the matrices $C^{(\alpha)}, D^{(\beta)}$ to within $m+n$ unitary matrices according to

$$
C^{(\alpha)} \longrightarrow C^{(\alpha)} U^{(\alpha)}
$$

which leave (11) unchanged but change the maps $B_{r r^{\prime}, s^{\prime} s}$.

\section{Dynamical Maps as Contractions:}

A straightforward way[4] of generating positive maps is to consider a unitary evolution of a coupled system. $R, S$ with $S$ being the system and $R$ a 'reservoir'. If we take a direct product density matrix

$$
\rho \rightarrow \rho \times \tau \rightarrow V \rho \times \tau V^{\dagger}
$$

where $V$ is a unitary matrix in the direct product space

$$
H_{m} \times H_{n}
$$


Then

$$
\rho_{r s} \times \tau_{a b} \rightarrow V_{r a, r^{\prime} a^{\prime}} \rho_{r^{\prime} s^{\prime}} \tau_{a^{\prime} b^{\prime}} V_{s b, s^{\prime} b}^{*}
$$

The partial trace operation is a contraction

$$
V \rho \times \tau V^{\dagger} \rightarrow V_{r n, r^{\prime} a^{\prime}} \rho_{r^{\prime} s^{\prime}} \tau_{a^{\prime} b^{\prime}} V_{s b, s^{\prime} b^{\prime}}
$$

If $\tau$ is made diagonal (if necessary by a unitary transformation in $H_{n}$ ) and the eigenvalues are $\tau_{(1)}, \tau_{(2)}, \ldots, \tau_{(n)}$, the map is

$$
\rho_{r s} \longrightarrow \sum_{\substack{\nu \\ r^{\prime} s^{\prime} \\ n}} V_{r r^{\prime}}(n, \nu, r) \rho_{r^{\prime} s^{\prime}} \tau(\nu) V_{s s^{\prime}}^{*}(n, \nu, \tau) .
$$

Clearly, if $\tau$ has more than one nonzero eigenvalue, the map is not extremal. So as far as extremal maps are concerned

$$
\rho_{r s} \longrightarrow \sum_{n} V_{r r^{\prime}}(n) \rho_{r^{\prime} s^{\prime}} V_{s s^{\prime}}^{*}(n)
$$

which is of the standard form [3]

$$
\rho \longrightarrow \sum_{\alpha} C^{(\alpha)} \rho C^{(\alpha) \dagger}
$$

in which $n^{\alpha}$ runs over at most $1 \leq n \leq m$. We note that all these maps are completely positive. We can also do an inverse reconstruction: given an extremal completely positive map

$$
\rho \longrightarrow \sum C^{(\alpha)} \rho C^{(\alpha) \dagger}
$$

we can define a reservoir matrix $\tau$ with all elements except $\tau_{11}$ zero and $\tau_{11}=1$. Then we can construct a unitary matrix $V$ in $m n$ dimensions with:

$$
V_{r \alpha, r^{\prime} 1}=C_{r r^{\prime}}(\alpha)
$$

The conditions on $C(\alpha)$ are transcribed into

$$
\sum_{\alpha} V_{r \alpha, r^{\prime}}, V_{s \alpha, s^{\prime}}^{*},=\delta_{r s}
$$


which is necessary for $V$ to be a unitary matrix. The ambiguity in constructing other elements does not affect the map. Thus a completely positive extremal map can always be obtained as a contraction of a unitary evolution[6]. The other elements $C^{(\alpha)}$ can be used to carry out the construction of the matrix $V$ and a generic diagonal matrix $\tau$.

\section{Not Completely Positive Maps as Contractions}

What about not completely positive maps? To obtain such a map by contraction we generalize the auxiliary space $H_{N}$ to be a space with an indefinite metric and $V$ to be a pseudounitary operator in the $M N$ dimensional space. Positivity is guaranteed if the generalized density matrix is entirely within the convex of positive metric states of the $M N$ dimensional space. Then the sum over $n$ in (26) goes over both positive and negative metric terms; but the resultant density matrix is nonnegative.

We can invert this derivation to realize the most general extremal not completely positive map as the contraction of a larger evolution in an indefinite metric space for the reservoir and a density matrix $\tau$ of the reservoir system to have a single eigenvector (with positive metric) with eigenvalue unity, the others being trivial.

Since such reservoirs are somewhat artificial, we may consider this reconstruction as a purely formal device. 


\section{Summary}

We have studied linear dynamical maps which take the set of density matrices into the set of density matrices. These maps form a convex set and are compact in the case of completely positive maps. The search for extremal maps gives us the restriction that we need at most $N$ terms for an extremal map. Those maps can be obtained as contractions of a direct product system: the extremal maps correspond to a reservoir matrix which is a projection. Conversely we can reconstruct the unitary evolution of the expanded system from the map itself.

The considerations are extended to positive but not completely positive dynamical maps. The extremal maps still contain at most $N$ terms. We can obtain these as contractions of an extended system with a pseudounitary evolution matrix. We could also reconstruct the extended pseudounitary evolution from the maps.

In the systematic parametrization we find we need $N(N-1) / 2$ parameters for the completely positive map apart from a set of unitary $N \times N$ matrices. For the corresponding parametrization of the not completely positive maps we have $\left(m^{2}+n^{2}\right)$ for $m$ positive and $n$ negative eigenvalues for the dynamical matrix and the unitary matrices (or less).

These results generalize the results obtained two decades ago by Gorini and Sudarshan[6] for $2 \times 2$ matrices.

Needless to say, however complicated the dynamical processes leading to the linear stochastic evolution that is represented by the dynamical map, we see that the same dynamics obtains with a reservoir having dimension $N^{2} \times N^{2}$. In the case of an extremal map it suffices to have a reservoir with a state space of the same $N \times N$ dimensional density matrices.

In this paper we have only dealt with dynamical maps, not the continuous semigroup of evolutions. This study was carried out by A. Kossakowski[9] and followed by others. [8,9] In these while the semigroup generators are parametrized no attempt is made to embed them in a larger system. Since the Zeno effect[10] operates for very small time intervals, care must be taken in generating a semigroup from the dynamics of an extended system. We hope to examine this question in the near future.

\section{References}

[1] E. C. G. Sudarshan, P. M. Mathews and J. Rau, "Stochastic Dynamics of Quantum Mechanical Systems", Phys. Rev. 121, 920-924 (1961).

[2] M. D. Choi, "Positive Real Maps on $C^{*}$ algebras", Can. J. Math. 24, 526 (1972); "On the Generators of Quantum Dynamical Semigroups," Illinois J. Math. 48, 119 (1976); E. B. Davies, "Quantum Theory of Open Systems," Academic Press (London) 1976. 
[3] E. C. G. Sudarshan, "Quantum Measurements and Dynamical Maps", in From $S U(3)$ to Gravity, ed. E. Gotsman and G. Tauber, Cambridge University Press (1986).

[4] The simplest not completely positive map is the transposition map $\rho \rightarrow \rho^{T}$.

[5] This was already recognized in reference 1 . See also reference 8.

[6] V. Gorini and E. C. G. Sudarshan, "Extreme Affine Transformations", Comm. Math. Phys. 46, 43 (1976).

[7] "Irreversibility and Dynamical Maps of Statistical Operators", Lecture Notes in Physics 29, 260, Springer Verlag, Berlin (1971).

[8] Goran Lindblad, "On the Generators of Quantum Dynamical Semigroups," Comm. Math. Phys. 48, 119 (1978).

[9] V. Gorini, A. Kossakowski and E. C. G. Sudarshan, "Properties of Quantum Markovian Master Equations," Rep. Math. Phys. 13, 149 (1978).

[10] B. Misra and E. C. G. Sudarshan, "The Zeno Paradox in Quantum Theory", J. Math. Phys. 18, 756 (1977). 